\title{
A Multilead Approach to T-Wave Alternans Detection Combining Principal Component Analysis and the Laplacian Likelihood Ratio Method
}

\author{
V Monasterio, JP Martínez \\ Comm Tech Group, Aragon Institute of Eng Research / CIBER-BBN, Univ of Zaragoza, Spain
}

\begin{abstract}
Several methods have been proposed to automatically detect and estimate T-wave alternans (TWA) in the ECG, all of them operating on a single-lead basis. In this study a multilead detection scheme is proposed. It combines Principal Component Analysis with the Laplacian Likelihood Ratio method to improve the detection results. A simulation is carried out to evaluate the effect of different types of simulated and physiological noise, and to assess the performance of this detection scheme. According to simulation results, the proposed multilead approach can detect alternans with a SNR $30 \mathrm{~dB}$ lower than the single-lead approach, showing a remarkable improvement of the sensitivity to low-level alternans.
\end{abstract}

\section{Introduction}

T-wave alternans (TWA) is defined as a consistent fluctuation in the repolarization morphology on an everyother-beat basis. TWA is presently regarded as a plausible index of susceptibility to sudden cardiac arrest [1]. Several methods exist to detect and estimate TWA. A comprehensive review can be found in [2]. In all of them TWA is detected on a single-lead basis. The main drawback of these methods is either their sensitivity to the presence of high amplitude nonalternant components, or their low sensitivity to low-level alternans $[1,2]$.

In this work, we propose a multilead approach that combines Principal Component Analysis (PCA) and the Laplacian Likelihood Ratio method (LLR) proposed in [3, 4]. With the proposed approach, the alternans information contained in different leads is concentrated in a subset of signals, discarding noise and artifacts. TWA analysis is applied considering only this subset of signals.

In this paper we evaluate the multilead approach and compare it with a single-lead detection scheme. In Sections 2 and 3, we describe the TWA analysis methods and the simulation study respectively. Simulation results are presented in Section 4. In Section 5 we expose the conclusions of this work.

\section{TWA analysis}

\subsection{Preprocessing}

In the preprocessing stage, the multilead input signal is low-pass filtered with a cut-off frequency of $60 \mathrm{~Hz}$, and decimated so that the resulting sampling frequency is 125 Hz. An example is shown in Figure 2.a. Let $L$ be the number of beats in the input signal, $N$ the number of samples of one ST-T complex, and $K$ the number of leads. For the $i$ :th beat, the ST-T complex $x_{i, k}(n)$ is extracted in leads $k=0 \ldots K-1$, and then complexes from the different leads are put together into a matrix $\mathbf{X}_{i}$

$$
\mathbf{X}_{i}=\left[\begin{array}{c}
\mathbf{x}_{i, 0}^{T} \\
\ldots \\
\mathbf{x}_{i, K-1}^{T}
\end{array}\right]=\left[\begin{array}{ccc}
x_{i, 0}(0) & \ldots & x_{i, 0}(N-1) \\
\ldots & \ldots & \ldots \\
x_{i, K-1}(0) & \ldots & x_{i, K-1}(N-1)
\end{array}\right]
$$

The $n$ :th column of $\mathbf{X}_{i}$ is formed by the amplitudes of the $K$ leads at a given time. The $\mathbf{X}_{i}$ matrices are concatenated to form the data matrix $\mathbb{X}$

$$
\mathbb{X}=\left[\begin{array}{llll}
\mathbf{X}_{0} & \mathbf{X}_{1} & \ldots & \mathbf{X}_{L-1}
\end{array}\right]
$$

The $k$ :th row of $\mathbb{X}$ contains the concatenated ST-T complexes corresponding to the $k$ :th lead.

\subsection{Single-lead approach}

In this approach, each lead is processed independently after the preprocessing stage. First, alternans detection is carried out lead by lead using the Generalized Likelihood Ratio Test for Laplacian noise (Laplacian GLRT) as proposed in $[3,4]$. To decide whether alternans is present or not, the resulting value of the test is compared to a threshold, which is calculated to obtain a fixed false alarm probability $\left(P_{F A}\right)$. The overall TWA test is positive if alternans is detected at least in one lead. Then, alternans estimation is carried out in each lead by computing the Maximum Likelihood Estimation for Laplacian noise (Laplacian MLE)[3, 4]. 


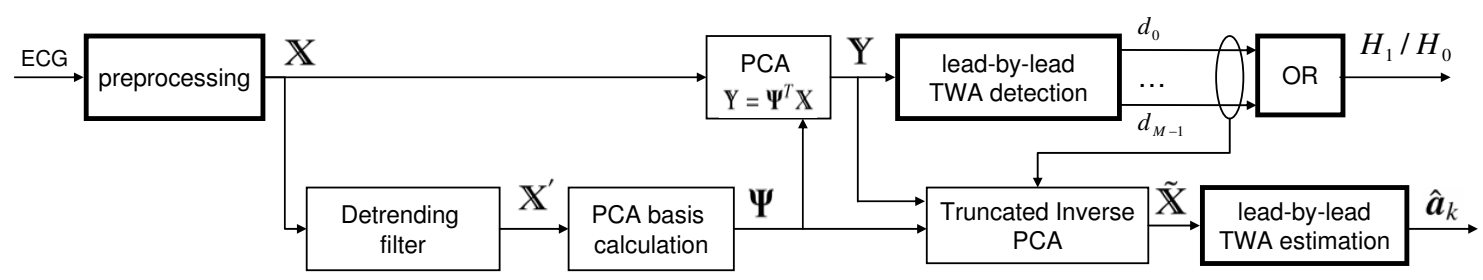

Figure 1. Block diagram of the multilead approach. The blocks in bold line are the ones used in the single-lead approach. Note that in the single-lead approach $\mathbf{Y}=\mathbb{X}=\tilde{\mathbf{X}}$.

\subsection{Multilead approach}

The block diagram of the proposed multilead detection and estimation process is given in Figure 1. After the preprocessing stage, a simple detrending filter is applied to the original data to eliminate the background ST-T complex

$$
\mathbf{x}_{i, k}^{\prime}=0.5\left(\mathbf{x}_{i, k}-\mathbf{x}_{i-1, k}\right), i=1 \ldots L-1
$$

A detrended data matrix $\mathbb{X}^{\prime}$ is defined as in (1) and (2) by piling leads and concatenating the detrended beats $\mathbf{x}_{i, k}^{\prime}$.

PCA is then applied based on the fact that the rows of $\mathbb{X}^{\prime}$ have zero mean. The interlead correlation matrix can be estimated as

$$
\hat{\mathbf{R}}_{\mathbb{X}^{\prime}}=\frac{1}{L N} \mathbb{X}^{\prime} \mathbb{X}^{\prime T}
$$

To obtain the whole set of $K$ principal components of $\mathbf{X}^{\prime}$, the eigenvector equation for $\hat{\mathbf{R}}_{\mathbf{X}^{\prime}}$ is solved

$$
\hat{\mathbf{R}}_{\mathbf{X}^{\prime}} \boldsymbol{\Psi}=\boldsymbol{\Psi} \boldsymbol{\Lambda}
$$

thus obtaining the eigenvectors matrix $\Psi$. Then, an orthonormal linear transformation based on $\boldsymbol{\Psi}$ is applied

$$
\mathbf{Y}=\boldsymbol{\Psi}^{T} \mathbb{X}
$$

to obtain the transformed data matrix Y. The $k$ :th row of $\mathrm{Y}$ contains the transformed data corresponding to the $k$ :th principal component, and from here on it will be referred to as the $k$ :th transformed lead for simplicity. Figure 2.b shows the transformed signal.

The main goal of PCA is to project the alternans information of $\mathbb{X}$ in a subset of components $M<K$, whereas noise is projected in the complementary subset. To choose this subset of components, TWA detection is carried out in each transformed lead by using the Laplacian GLRT as proposed in [3,4], where a statistic $Z$ is computed from the data and compared to a threshold. The result of this leadby-lead detection is denoted as $d_{k}$ : if alternans is detected in the $k$ :th transformed lead, $d_{k}=1$, and $d_{k}=0$ otherwise. The overall TWA test is positive if alternans is detected at least in one transformed lead.

For the TWA estimation stage, both principal components and transformed leads corresponding with $k$ such that $d_{k}=0$ are eliminated from $\boldsymbol{\Psi}$ and $\mathbf{Y}$, thus obtaining the truncated basis matrix $\boldsymbol{\Psi}_{t r}$ and the truncated transformed data matrix $\mathbf{Y}_{t r}$ respectively. A new signal in the original domain is then reconstructed with the truncated matrices,

$$
\tilde{\mathbf{X}}=\boldsymbol{\Psi}_{t r} \mathbf{Y}_{t r}
$$

Figure 2.c shows the reconstructed signal. Note that $\tilde{\mathbf{X}}$ is equivalent to a spatially filtered signal, where the equivalent filter is aimed at preserving the alternans content, not at obtaining a perfect reconstruction of the original signal. The reconstructed data matrix $\tilde{\mathbf{X}}$ consists of the concatenation of the multilead single-beat matrices $\tilde{\mathbf{X}}_{i}$ :

$$
\tilde{\mathbf{X}}=\left[\begin{array}{llll}
\tilde{\mathbf{X}}_{0} & \tilde{\mathbf{X}}_{1} & \ldots & \tilde{\mathbf{X}}_{L-1}
\end{array}\right]
$$

where

$$
\tilde{\mathbf{X}}_{i}=\left[\begin{array}{c}
\tilde{\mathbf{x}}_{i, 0}^{T} \\
\ldots \\
\tilde{\mathbf{x}}_{i, K-1}^{T}
\end{array}\right]
$$

with $\tilde{\mathbf{x}}_{i, k}$ corresponding to the reconstructed ST-T segment of the $i$ :th beat in the $k$ :th lead. Laplacian MLE is then applied to the reconstructed data as proposed in $[3,4]$ to estimate the alternans waveform and amplitude. Figure 2.d shows an example of estimated alternans.

\section{Simulation study}

A simulation study was carried out to evaluate the multilead approach and to compare it with the single-lead scheme. Simulation setup is shown in Figure 3. We simulated multilead ECG signals by adding noise and TWA to a clean background ECG. Only the eight independent leads V1 to V6, I and II were considered $(K=8)$. For the background ECG we selected a standard beat from a 12-lead record and repeated it $L=32$ times. The alternans waveform was estimated and extracted from another 12-lead record, using the LLR method as described in [4]. Both records belonged to the STAFF-III database [4].

We considered four sources of noise: Gaussian $(g s)$, Laplacian $(l p)$, electrode motion $(\mathrm{em})$ and muscular activity $(\mathrm{ma})$. Physiological noises $\mathrm{em}$ and $m a$ were extracted from the MIT-BIH Noise Stress Test Database [5]. A description of their statistics can be found in [3]. For each realization, $K$ noise segments of $L \times N$ samples were simulated ( $g s$ or $l p$ noise) or extracted from the records beginning at a random position (em or $m a$ noise). Baseline wander was removed from $\mathrm{em}$ and $\mathrm{ma}$ noises. Each segment was normalized so that its RMS value was $1 \mu V$.

Let $\mathbf{W}$ be the multilead noise matrix formed by the noise segments $\mathbf{w}_{k}$, 


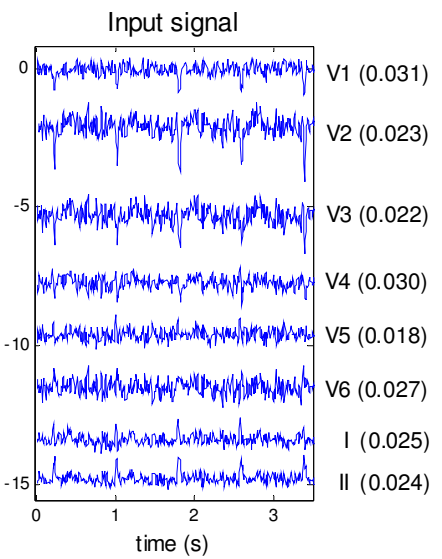

(a)

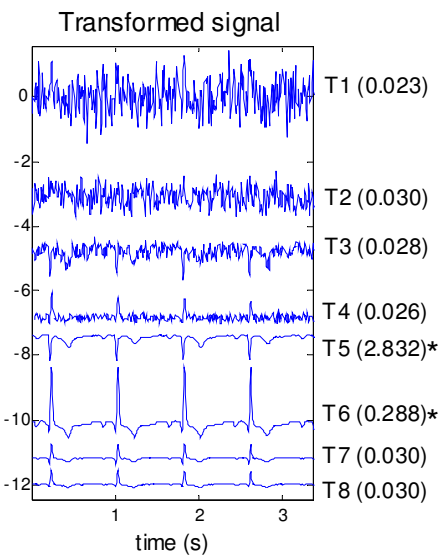

(b)

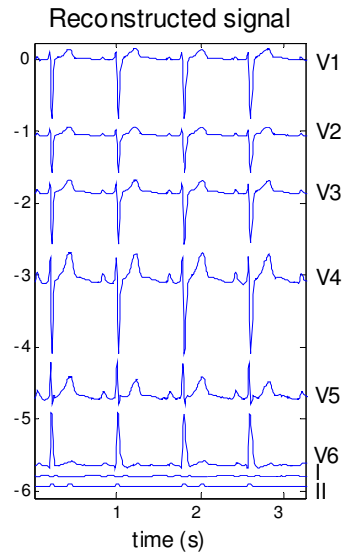

(c)

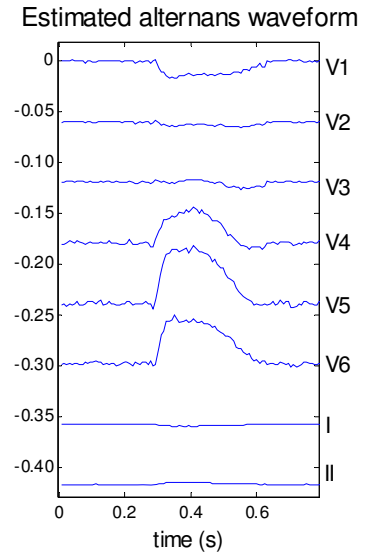

(d)

Figure 2. (a) Input signal with SNR $=-20 \mathrm{~dB}$. (b) Transformed signal after PCA. (c) Reconstructed signal after truncated inverse PCA. (d) Estimated alternans waveform. The GLRT values for single-lead and multilead approaches are shown in brackets in (a) and (b) respectively. An asterisk indicates the leads that overcome the detection threshold (T5 and T6). Note that alternans are visible in T5, T6, and V4 to V6 in the reconstructed signal. Ordinate units are in mV.

$$
\mathbf{W}=\left[\begin{array}{c}
\mathbf{w}_{0}^{T} \\
\ldots \\
\mathbf{w}_{K-1}^{T}
\end{array}\right]
$$

it can be assumed that segments are mutually uncorrelated, i.e.

$$
\hat{\mathbf{R}}_{W}=\frac{1}{N L} \mathbf{W} \mathbf{W}^{T}=\mathbb{I}
$$

However, in real ECG records the noise in the different leads is not uncorrelated. To simulate a physiological-like correlation, we selected 2000 segments of noise for each lead from 10 records of the PTB Diagnostic ECG database [6]. For each segment, the $50 \mathrm{~ms}$ interval prior to a $\mathrm{P}$ wave onset was selected, and DC level was removed. Segments corresponding to each lead were concatenated, and the resulting noise leads were piled similarly to (10) to form a noise matrix $\mathbf{N}$. The interlead correlation matrix of $\mathbf{N}$ was estimated similarly to (11). Its inverse was decomposed as

$$
\hat{\mathbf{R}}_{N}^{-1}=\mathbf{D D}^{T}
$$

where $\mathbf{D}$ is an upper triangular matrix with strictly positive

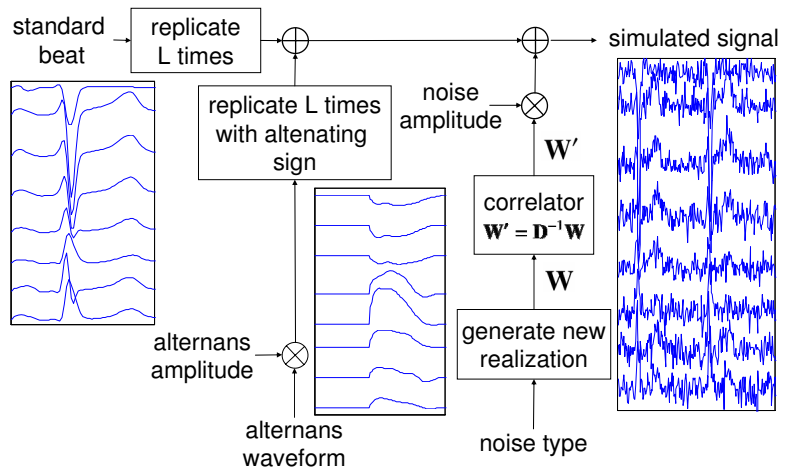

Figure 3. Simulation of ECG signals with TWA and noise. Signals scale is not preserved for better visualization. diagonal entries. The inverse of $\mathbf{D}$ was used to spatially correlate the generated noise

$$
\mathbf{W}^{\prime}=\mathbf{D}^{-1} \mathbf{W}
$$

thus obtaining a noise matrix $\mathbf{W}^{\prime}$ whose interlead correlation equals $\hat{\mathbf{R}}_{N}$. Finally, the noise was scaled so that the RMS value of the least noisy lead was $200 \mu V$. Alternans was then scaled to obtain a desired value of SNR, defined as the ratio between alternans power and noise power.

\section{Results}

We simulated ECG fragments with the four types of noise described in Section 3, with SNR values ranging from -60 to $10 \mathrm{~dB}$, and also without TWA. For each parameter combination, $10^{4}$ noise realizations were generated, and signals were processed with both the single-lead and the multilead methods.

Figure 4 shows the evolution of the GLRT statistic $Z$ in the eight transformed leads versus different levels of SNR. Each point represents the average $Z$ value of the $10^{4}$ realizations. We found that when SNR $\leq-30 \mathrm{~dB}$, alternans content was mainly projected in the 5th transformed lead (T5); later, as SNR rose to $-10 \mathrm{~dB}$, alternans appeared also in T6, T7 and T8 successively. When SNR $\geq-5 \mathrm{~dB}$, it appeared predominantly in $\mathrm{T} 1, \mathrm{~T} 2$ and $\mathrm{T} 3$. These results were similar for all the noises.

Receiver Operating Characteristic curves (ROC curves) were computed by sweeping different threshold values. Figure 5 shows the curves of the two methods corresponding to $\mathrm{SNR}=-45 \mathrm{~dB}$. To analyze the behavior of the methods for different values of SNR, we selected a fixed value for the threshold so that $P_{F A}=0.01$, and compared the resulting detection probability $P_{D}$. In Figure $6, P_{D}$ for $P_{F A}=0.01$ is plotted against SNR for the different types of noise and for the two methods. 


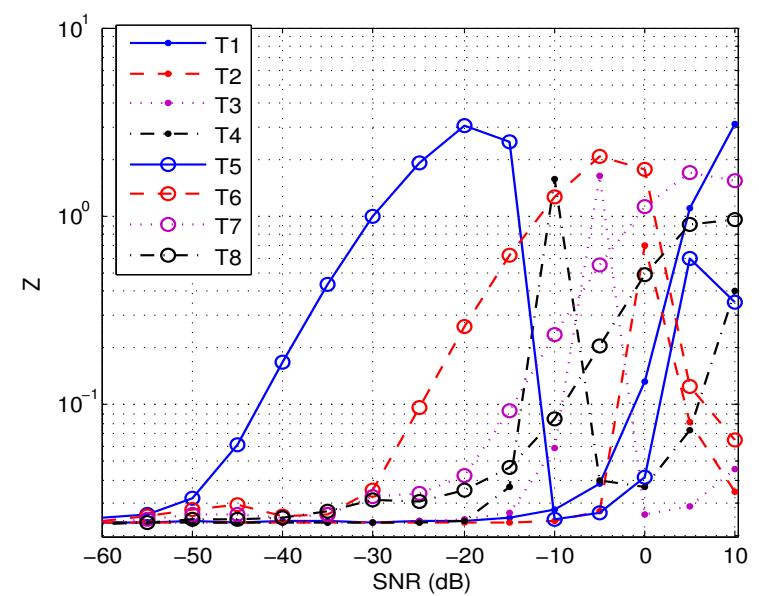

Figure 4. Evolution of the statistic in the transformed leads vs. SNR for Gaussian noise.
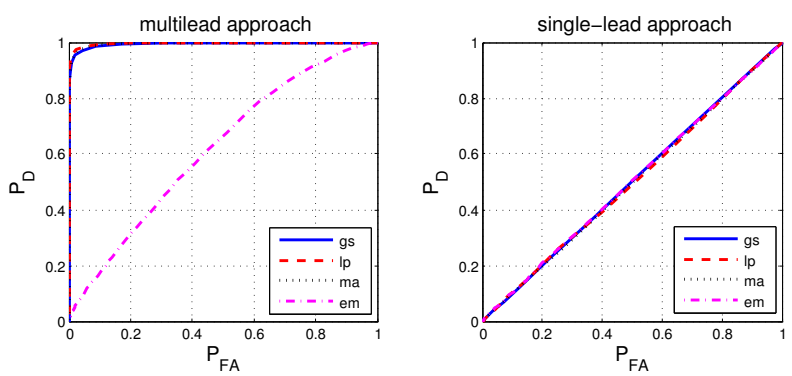

Figure 5. ROC curves for $\mathrm{SNR}=-45 \mathrm{~dB}$

\section{Discussion and conclusions}

The high performance of the multilead method is similar for $g s, l p$ and $m a$ noises, as shown in Figure 5. It is worse when facing the $\mathrm{em}$ noise, because $\mathrm{em}$ bandwidth mostly overlaps the band of the alternans. Even in this case, the multilead approach performs better than the single-lead approach. Note that in Figure 5 the single-lead method is not capable of detecting anything due to the low SNR level.

As we can see in Figure 6, the multilead method surpasses widely the performance of the single-lead method. In the worst case, for a fixed value of $P_{D}$ the multilead method can detect alternans with a SNR $30 \mathrm{~dB}$ lower than the single-lead method. The improvement of the performance is similar for the different types of noise. The multilead scheme performs better, specially at low SNR levels, because the method discards most of the noise. For instance, at $\mathrm{SNR}=-20 \mathrm{~dB}$, alternans is projected in only two leads (see Figure 2), and the noise contained in the rest is totally discarded, allowing a more precise TWA analysis.

Simulation results suggest that the multilead approach can improve significantly the sensitivity to low level alternans when standard 12-lead ECG is available. The proposed scheme may find application in noisy signals such as stress test ECG.
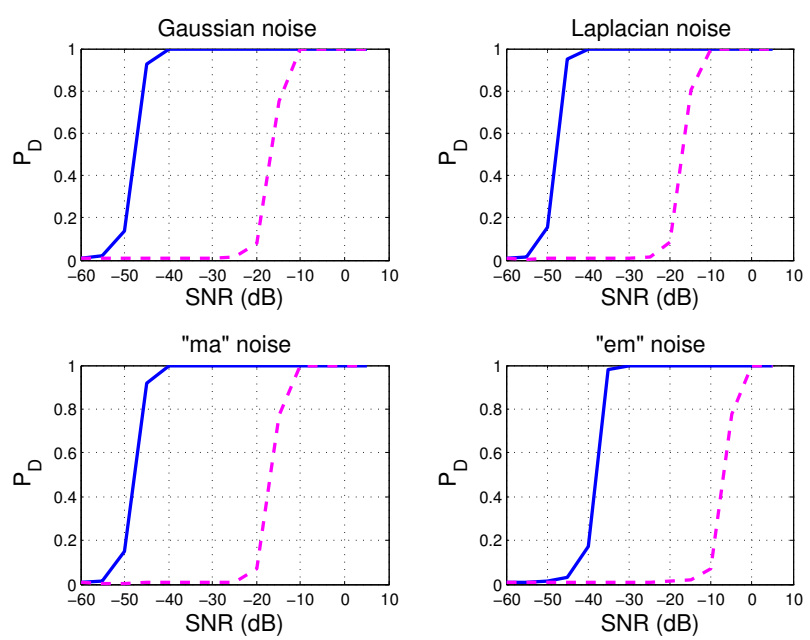

Figure 6. $P_{D}$ for $P_{F A}=0.01$ of the multilead (solid line) and the single-lead approach (dashed line) vs. SNR.

\section{Acknowledgements}

This work was supported by projects TEC-2004-05263C02 from CICYT, PI04/0689 from FIS and GTC T-30 from DGA (Spain).

\section{References}

[1] Narayan SM. T-Wave Alternans and the Susceptibility to Ventricular Arrhythmias. J Am Coll Cardiol 2006; 47(2):269-281.

[2] Martínez JP, Olmos S. Methodological principles of T wave alternans analysis: a unified framework. IEEE Trans Biomed Eng 2005;52:599-613.

[3] Martínez JP, Olmos S. A robust T-wave alternans detector based on the glrt for laplacian noise distribution. In Computers in Cardiology 2002, volume 29. IEEE Comp. Soc. Press, 2002; 677-680.

[4] Martínez JP, Olmos S, Wagner G, Laguna P. Characterization of repolarization alternans during ischemia: time-course and spatial analysis. IEEE Trans Biomed Eng 2006;53:701-711.

[5] Moody GB, Mark RG. The MIT-BIH arrhythmia database on CD-ROM and software for use with it. In Computers in Cardiology 1990. IEEE Comp. Soc. Press, 1990; 185-188.

[6] Bousseljot R, Kreiseler D, Schnabel A. Nutzung der EKGSignaldatenbank CARDIODAT der PTB über das internet. Biomedizinische Technik 1995;40:317-318.

Address for correspondence:

Violeta Monasterio Bazán

Dep. Ingeniería Electrónica y Comunicaciones

María de Luna,3. 50018, Zaragoza, Spain

violeta.monasterio@unizar.es 